Random geometric graph diameter in the unit ball (with Robert B. Ellis and Catherine Yan) Algorithmica 47, no. 4 (2007), $421--438$. The original publication is available at www.springerlink.com: http://dx.doi.org/10.1007/s00453-006-0172-y Open Access version: http://kuscholarworks.ku.edu/dspace/.

[This document contains the author's accepted manuscript. For the publisher's version, see the link in the header of this document.]

\title{
RANDOM GEOMETRIC GRAPH DIAMETER IN THE UNIT BALL
}

\author{
ROBERT B. ELLIS, JEREMY L. MARTIN, AND CATHERINE YAN
}

\begin{abstract}
The unit ball random geometric graph $G=G_{p}^{d}(\lambda, n)$ has as its vertices $n$ points distributed independently and uniformly in the unit ball in $\mathbb{R}^{d}$, with two vertices adjacent if and only if their $\ell_{p}$-distance is at most $\lambda$. Like its cousin the Erdős-Rényi random graph, $G$ has a connectivity threshold: an asymptotic value for $\lambda$ in terms of $n$, above which $G$ is connected and below which $G$ is disconnected. In the connected zone, we determine upper and lower bounds for the graph diameter of $G$. Specifically, almost always, $\operatorname{diam}_{p}(\mathbf{B})(1-$ $o(1)) / \lambda \leq \operatorname{diam}(G) \leq \operatorname{diam}_{p}(\mathbf{B})\left(1+O\left((\ln \ln n / \ln n)^{1 / d}\right)\right) / \lambda$, where $\operatorname{diam}_{p}(\mathbf{B})$ is the $\ell_{p}$-diameter of the unit ball $\mathbf{B}$. We employ a combination of methods from probabilistic combinatorics and stochastic geometry.
\end{abstract}

\section{INTRODUCTION}

A random geometric graph consists of a set of vertices distributed randomly over some metric space $X$, with two vertices joined by an edge if the distance between them is sufficiently small. This construction presents a natural alternative to the classical Erdős-Rényi random graph model, in which the presence of each edge is an independent event (see, e.g., [2]). The study of random geometric graphs is a relatively new area; the monograph [9] by M. Penrose is the current authority. In addition to their theoretical interest, random geometric graphs have many applications, including wireless communication networks; see, e.g., [4, 10, 12].

In this article, we study the unit ball random geometric graph $G=G_{p}^{d}(\lambda, n)$, defined as follows. Let $d$ and $n$ be positive integers, $\mathbf{B}$ the Euclidean unit ball in $\mathbb{R}^{d}$ centered at the origin, $\lambda$ a positive real number, and $p \in[1, \infty]$ (that is, either $p \in[1, \infty)$ or $p=\infty)$. Let $V_{n}$ be a set of $n$ points in $\mathbf{B}$, distributed independently and uniformly with respect to Lebesgue measure on $\mathbb{R}^{d}$. Then $G$ is the graph with vertex set $V_{n}$, where two vertices $x=\left(x_{1}, \ldots, x_{d}\right)$ and $y=\left(y_{1}, \ldots, y_{d}\right)$ are adjacent if and only if $\|x-y\|_{p} \leq \lambda$. (Thus the larger $\lambda$ is, the more edges $G$ has.) Here $\|\cdot\|_{p}$ is the $\ell_{p}$-metric defined by

$$
\|x-y\|_{p}= \begin{cases}\left(\sum_{i=1}^{d}\left|x_{i}-y_{i}\right|^{p}\right)^{1 / p} & \text { for } p \in[1, \infty), \\ \max \left\{\left|x_{i}-y_{i}\right|: 1 \leq i \leq d\right\} & \text { for } p=\infty\end{cases}
$$

where the case $p=2$ gives the standard Euclidean metric on $\mathbb{R}^{d}$.

When $d=1, G$ is known as a random interval graph. (Note that the value of $p$ is immaterial when $d=1$.) Random interval graphs have been studied extensively in the literature; the asymptotic distributions for the number of isolated vertices and the number of connected components were determined precisely by E. Godehardt and J. Jaworski [7]. The random Euclidean unit disk graph $G_{2}^{2}(\lambda, n)$ was studied by X. Jia and the first and third authors [5]. 
Random geometric graph diameter in the unit ball (with Robert B. Ellis and Catherine Yan) Algorithmica 47, no. 4 (2007), $421--438$. The original publication is available at www.springerlink.com: http://dx.doi.org/10.1007/s00453-006-0172-y Open Access version: http://kuscholarworks.ku.edu/dspace/.

In the present article, we focus on the case $d \geq 2$ and $p \in[1, \infty]$, but also comment along the way on the special case $d=1$. We are interested in the asymptotic behavior of the connectivity and graph diameter of $G$ as $n \rightarrow \infty$ and $\lambda \rightarrow 0$. In fact, $G$ has a connectivity threshold: roughly speaking, an expression for $\lambda$ as a function of $n$, above which $G$ is connected and below which $G$ is disconnected. (This behavior is ubiquitous in the theory of the Erdős-Rényi random graph model; cf. [2].)

We are interested primarily in the combinatorial graph diameter of $G, \operatorname{diam}(G)$, above the connectivity threshold. Our results include

- a lower bound for $\operatorname{diam}(G)$ (Proposition 7 of $\S 4$ );

- an "absolute" upper bound $\operatorname{diam}(G)<K / \lambda$, where $K$ is a constant depending only on $d$ (Theorem 8 of $\S 5$ ); and

- an asymptotically tight upper bound within a factor of the form $(1+o(1))$ of the lower bound, the proof of which builds on the absolute upper bound (Theorem 10 of $\S 6$ ).

\section{Definitions and Notation}

As mentioned above, the main object of our study is the random geometric graph $G=G_{p}^{d}(\lambda, n)$, where $d \geq 1$ is the dimension of the ambient unit ball $\mathbf{B}$, $p \in[1, \infty]$ describes the metric, $\lambda>0$ is the $\ell_{p}$-distance determining adjacency, and $n$ is the number of vertices. We will generally avoid repeating the constraints on the parameters.

The graph distance $d_{G}(x, y)$ between two vertices $x, y \in V_{n}$ is defined to be the length of the shortest path between $x$ and $y$ in $G$, or $\infty$ if there is no such path. The graph diameter of $G$ is defined to be $\operatorname{diam}(G):=\max \left\{d_{G}(x, y): x, y \in V_{n}\right\}$. This graph-theoretic quantity is not to be confused with the $\ell_{p}$-diameter of a set $X \subseteq \mathbb{R}^{d}$, defined as $\operatorname{diam}_{p}(X):=\sup \left\{\|x-y\|_{p}: x, y \in X\right\}$. The $\ell_{p}$-ball of radius $r$ centered at $x \in \mathbb{R}^{d}$ is defined as

$$
B_{p}^{d}(x, r):=\left\{y \in \mathbb{R}^{d}:\|x-y\|_{p} \leq r\right\},
$$

while the $\ell_{p}$-ball of radius $r$ around a set $X \subseteq \mathbb{R}^{d}$ is $B_{p}^{d}(X, r):=\cup_{x \in X} B_{p}^{d}(x, r)$. The origin of $\mathbb{R}^{d}$ is $O:=(0, \ldots, 0)$; when the center of a ball is not explicitly given, we define $B_{p}^{d}(r):=B_{p}^{d}(O, r)$. Thus $\mathbf{B}=B_{2}^{d}(1)$. The $\ell_{p}$-diameter of $\mathbf{B}$ is

$$
\operatorname{diam}_{p}(\mathbf{B})= \begin{cases}2 d^{1 / p-1 / 2} & \text { when } 1 \leq p \leq 2, \\ 2 & \text { when } 2 \leq p \leq \infty\end{cases}
$$

The distance $d_{p}(X, Y)$ between two sets $X, Y \subseteq \mathbb{R}^{d}$ is defined as inf $\left\{\|x-y\|_{p}: x \in\right.$ $X, y \in Y\}$. The boundary $\partial X$ of $X$ is its closure minus its interior (in the usual topology on $\left.\mathbb{R}^{d}\right)$, and its volume $\operatorname{vol}(X)$ is its Lebesgue measure.

We will make frequent use of the quantity

$$
\alpha_{p}^{d}:=\frac{\operatorname{vol}\left(B_{p}^{d}(r)\right)}{\operatorname{vol}\left(B_{2}^{d}(r)\right)}=\frac{\Gamma\left(\frac{p+1}{p}\right)^{d} \cdot \Gamma\left(\frac{2+d}{2}\right)}{\Gamma\left(\frac{3}{2}\right)^{d} \cdot \Gamma\left(\frac{p+d}{p}\right)},
$$

where $\Gamma$ is the usual gamma function (see, e.g., [11]). The calculation of $\alpha_{p}^{d}$, along with the proofs of several other useful facts about $\ell_{p}$-geometry, may be found in the Appendix at the end of the article. 
Random geometric graph diameter in the unit ball (with Robert B. Ellis and Catherine Yan) Algorithmica 47, no. 4 (2007), $421--438$. The original publication is available at www.springerlink.com: http://dx.doi.org/10.1007/s00453-006-0172-y Open Access version: http://kuscholarworks.ku.edu/dspace/.

We will say that the random graph $G$ has a property $P$ almost always, or a.a., if

$$
\lim _{n \rightarrow \infty} \operatorname{Pr}[G \text { has property } P]=1 \text {. }
$$

By the notation $f(n)=o(g(n))$ and $f(n)=O(g(n))$, we mean, respectively, $\lim _{n \rightarrow \infty} f(n) / g(n)=0$ and $\limsup _{n \rightarrow \infty} f(n) / g(n) \leq c$, for some absolute nonnegative constant $c$.

\section{Connectivity thresholds}

In order for $G=G_{p}^{d}(\lambda, n)$ to have finite diameter, it must be connected. Therefore, we seek a connectivity threshold - a lower bound on $\lambda$ so that $G$ is almost always connected. When $d=1$, all $\ell_{p}$-metrics are identical. For this case we now quote parts of Theorems 10 and 12 of [7], to which we refer the reader for their precise determination of the asymptotic Poisson distributions of the number of isolated vertices and the number of connected components.

Theorem 1 (Godehardt, Jaworski). Let $\lambda_{1}(n)=\frac{1}{n}(\log n+c+o(1))$ and $\lambda_{2}(n)=$ $\frac{2}{n}(\log n+c+o(1))$, where $c$ is a constant. Then

$$
\begin{aligned}
\lim _{n \rightarrow \infty} \operatorname{Pr}\left[G_{p}^{1}\left(\lambda_{1}(n), n\right) \text { has an isolated vertex }\right] & =e^{-e^{-c}}, \\
\lim _{n \rightarrow \infty} \operatorname{Pr}\left[G_{p}^{1}\left(\lambda_{2}(n), n\right) \text { is connected }\right] & =e^{-e^{-c}} .
\end{aligned}
$$

In particular, by replacing $c$ in Theorem 1 with a nonnegative sequence $\gamma(n) \rightarrow$ $\infty$, almost always $G_{p}^{1}\left(\lambda_{1}(n), n\right)$ has no isolated vertices and $G_{p}^{1}\left(\lambda_{2}(n), n\right)$ is connected. The case $d=1$ is exceptional in that the thresholds for having isolated vertices and for connectivity are separated.

For $d \geq 2$, we will use the fact that the connectivity threshold coincides with the threshold for the disappearance of isolated vertices, which follows from two theorems of M. Penrose. First we compute the threshold for isolated vertices, which is easier to calculate.

Proposition 2. Let $d \geq 2$, let $p \in[1, \infty]$, and let $\alpha=\alpha_{p}^{d}$ be the constant of (1). Suppose $\gamma(n)$ is a nonnegative sequence such that $\lim _{n \rightarrow \infty} \gamma(n) \rightarrow \infty$, and that

$$
\lambda \geq\left(\frac{1}{\alpha n}\left(\frac{2(d-1)}{d} \ln n+\frac{2}{d} \ln \ln n+\gamma(n)\right)\right)^{1 / d} .
$$

Then, almost always, $G=G_{p}^{d}(\lambda, n)$ has no isolated vertices.

Proof. Let $V_{n}=\left\{v_{1}, v_{2}, \ldots, v_{n}\right\}$ be the vertex set of $G$. For each vertex $v_{i}$, let $A_{i}$ be the event that $v_{i}$ is an isolated vertex, and let $X_{i}$ be the indicator of $A_{i}$; that is, $X_{i}=1$ if $A_{i}$ occurs and 0 otherwise. Set $X=X_{1}+X_{2}+\cdots+X_{n}$. We will show that $\mathbb{E}[X]=o(1)$.

By definition, $v_{i}$ is isolated if and only if there are no other vertices in $B_{p}^{d}\left(v_{i}, \lambda\right) \cap$ B. We condition $\operatorname{Pr}\left[A_{i}\right]$ on the $\ell_{2}$-distance from $v_{i}$ to the origin $O$. If $\left\|v_{i}-O\right\|_{2} \in$ $\left[0,1-d^{1 / 2} \lambda\right)$, then $B_{p}^{d}\left(v_{i}, \lambda\right) \subseteq \mathbf{B}$. Otherwise, if $\left\|v_{i}-O\right\|_{2} \in\left(1-d^{1 / 2} \lambda, 1\right]$, then the volume of $B_{p}^{d}\left(v_{i}, \lambda\right) \cap \mathbf{B}$ is not less than $\frac{1}{2} \operatorname{vol}\left(B_{p}^{d}\left(v_{i}, \lambda\right)\right)(1+O(\lambda))$. Hence

$$
\begin{aligned}
\operatorname{Pr}\left[A_{i}\right] \leq & \left(1-d^{1 / 2} \lambda\right)^{d}\left(1-\alpha \lambda^{d}\right)^{n-1}+ \\
& \left(1-\left(1-d^{1 / 2} \lambda\right)^{d}\right)\left(1-\frac{\alpha \lambda^{d}}{2}(1+O(\lambda))\right)^{n-1} .
\end{aligned}
$$


Random geometric graph diameter in the unit ball (with Robert B. Ellis and Catherine Yan) Algorithmica 47, no. 4 (2007), $421--438$. The original publication is available at www.springerlink.com: http://dx.doi.org/10.1007/s00453-006-0172-y Open Access version: http://kuscholarworks.ku.edu/dspace/.

Using $1-x=e^{-x}(1+o(1))$ as $x \rightarrow 0$ and the binomial expansion, we have

$$
\operatorname{Pr}\left[A_{i}\right] \leq(1+o(1)) e^{-\alpha n \lambda^{d}}+\left(d d^{1 / 2} \lambda+O\left(\lambda^{2}\right)\right)(1+o(1)) e^{-\alpha n \lambda^{d} / 2} .
$$

The first term is $o\left(n^{-1}\right)$ for $d \geq 2$. By linearity of expectation, $\mathbb{E}[X]=n \cdot \operatorname{Pr}\left[A_{i}\right]$, and so

$$
\mathbb{E}[X] \leq o(1)+d^{3 / 2} n \lambda(1+o(1)) n^{-1+1 / d}(\ln n)^{-1 / d} e^{-\gamma(n) / 2} .
$$

The second term is $o(1)$, and so $\operatorname{Pr}[X>0] \leq \mathbb{E}[X] \leq o(1)$; that is, almost always, $G$ has no isolated vertices.

The number of isolated vertices below the threshold is easy to compute in certain special cases. For example, if $p \in[1, \infty], d=2, \lambda=\sqrt{c \ln n / n}$, and $0 \leq c<\alpha^{-1}$, then a minor modification of [5, Theorem 1] yields $X=(1+o(1)) n^{1-\alpha c}$ almost always. In general, to determine the behavior of $X$ more exactly would require complicated integrals that describe the volume of $B_{p}^{d}\left(v_{i}, \lambda\right) \cap \mathbf{B}$ near the boundary of $\mathbf{B}$ (cf. [9, Chapter 8]). For our purposes, it suffices to concentrate on the values of $\lambda$ for which $G$ has no isolated vertices.

For $d \geq 2$ and $p \in(1, \infty]$, the connectivity threshold for the unit-cube random geometric graph coincides with the threshold for lacking isolated vertices. We quote Penrose's theorem [8, Thm. 1.1] after some supporting definitions. Define the unit cube geometric graph $H=H_{p}^{d}(\lambda, n)$ analogously to $G=G_{p}^{d}(\lambda, n)$, except that its vertices are points in $[0,1]^{d}$ rather than $\mathbf{B}$. For any nonnegative integer $k$, define

$$
\begin{aligned}
& \rho(H ; \kappa \geq k+1)=\min \{\lambda \mid H \text { has vertex connectivity } \kappa \geq k+1\}, \\
& \rho(H ; \delta \geq k+1)=\min \{\lambda \mid H \text { has minimum degree } \delta \geq k+1\} .
\end{aligned}
$$

Theorem 3 (Penrose). Let $p \in(1, \infty]$ and let $k \geq 0$ be an integer. Then

$$
\lim _{n \rightarrow \infty} \operatorname{Pr}[\rho(H ; \kappa \geq k+1)=\rho(H ; \delta \geq k+1)]=1 .
$$

When $k=0$, Theorem 3 asserts that as $\lambda$ increases (forcing more edges into the graph), almost always, $H$ becomes connected simultaneously as the last isolated vertex disappears. In the proof of Theorem 3 in [8], Penrose shows that the limiting probability distributions for $\rho(H ; \kappa \geq k+1)$ and $\rho(H ; \delta \geq k+1)$ are the same. The proof requires only a series of geometric and probabilistic arguments which hold in the unit ball as well as in the unit cube (see, in particular, Sections 2 and 5 of [8]), so we have as an immediate corollary the following.

Corollary 4. Let $d \geq 2$ and $p \in(1, \infty]$, and let $\lambda=\lambda(n)$ be sufficiently large so that, almost always, $G=G_{p}^{d}(\lambda(n), n)$ has no isolated vertices. Then, almost always, $G$ is connected.

We now consider the case that $d \geq 2$ and $p=1$. Here Theorem 3 does not apply. However, we can appeal to two general results about the behavior of a random geometric graph in an $\ell_{p}$-metric space whose boundary is a compact $(d-1)$ submanifold of $\mathbb{R}^{d}$ For such a graph, Theorem 7.2 of [9] provides a threshold for the disappearance of isolated vertices, and Theorem 13.7 provides a threshold for connectivity. Applying these results to $G$, with the thresholds for $G$ defined as in (2a) and (2b), we obtain the following fact. 
Random geometric graph diameter in the unit ball (with Robert B. Ellis and Catherine Yan) Algorithmica 47, no. 4 (2007), $421--438$. The original publication is available at www.springerlink.com: http://dx.doi.org/10.1007/s00453-006-0172-y Open Access version: http://kuscholarworks.ku.edu/dspace/.

Proposition 5. Let $d \geq 2, p \in[1, \infty]$, and $G=G_{p}^{d}(\lambda(n), n)$. Let $\alpha=\alpha_{p}^{d}$ be the constant of (1), and let $k \geq 0$ be an integer. Then, almost always,

$$
\lim _{n \rightarrow \infty}\left(\frac{n \alpha}{\log n} \rho(G ; \kappa \geq k+1)^{d}\right)=\lim _{n \rightarrow \infty}\left(\frac{n \alpha}{\log n} \rho(G ; \delta \geq k+1)^{d}\right)=\frac{2(d-1)}{d} .
$$

We now collect the above results to present the connectivity thresholds that we will use in the rest of the paper.

Theorem 6. Let $G=G_{p}^{d}(\lambda, n)$ and let $\alpha=\alpha_{p}^{d}$ be the constant of (1).

(i) Suppose that $d \geq 2, p \in(1, \infty], \gamma(n)$ is a nonnegative sequence such that $\gamma(n) \rightarrow \infty$ and

$$
\lambda \geq\left(\frac{1}{\alpha n}\left(\frac{2(d-1)}{d} \ln n+\frac{2}{d} \ln \ln n+\gamma(n)\right)\right)^{1 / d} .
$$

Then, almost always, $G$ is connected.

(ii) Suppose that $d \geq 2, p \in[1, \infty]$, and $\lambda=(c \ln n / n)^{1 / d}$ for some constant $c>0$. Then, almost always, $G$ is connected if $c>(2(d-1) /(d \alpha))$, and disconnected if $c<(2(d-1) /(d \alpha))$.

(iii) Suppose that $d=1, p \in[1, \infty]$, and $\lambda=2(\ln n+\gamma(n)) / n$. Then, almost always, $G$ is connected if $\gamma(n) \rightarrow \infty$ and disconnected if $\gamma(n) \rightarrow-\infty$.

Assertion (i) follows from combining Proposition 2 with Corollary 4, and assertion (ii) is implied by Proposition 5. (When $p>1$ and $d \geq 2$, the lower bound in (ii) is implied by the stronger bound in (i).) Assertion (iii) is implied by Theorem 1 and its accompanying remarks.

\section{A LOWER BOUND FOR DIAMETER}

When $G$ is connected, $\mathbf{B}$ will usually contain two vertices whose $\ell_{p}$-distance is (asymptotically) $\operatorname{diam}_{p}(\mathbf{B})$. Therefore, the diameter of $G$ will almost always be at least $\operatorname{diam}_{p}(\mathbf{B})(1-o(1)) / \lambda$. The precise statement is as follows.

Proposition 7 (Diameter lower bound). Let $d \geq 1$ and $p \in[1, \infty]$, and suppose that $\lambda=\lambda(n)$ is sufficiently large so that Theorem 6 guarantees that almost always, $G=G_{p}^{d}(\lambda, n)$ is connected. If $h=h(n)$ satisfies

$$
\lim _{n \rightarrow \infty} h^{(d+1) / 2} n=\infty,
$$

then, almost always,

$$
\operatorname{diam}(G) \geq \frac{1-h}{\lambda} \operatorname{diam}_{p}(\mathbf{B})= \begin{cases}2(1-h) d^{1 / p-1 / 2} / \lambda & \text { if } p \leq 2 \\ 2(1-h) / \lambda & \text { if } p \geq 2\end{cases}
$$

Proof. Let $\pm a$ be a pair of antipodes of the unit ball $\mathbf{B}$, chosen as in Figure 4, and let $\pm C$ be the spherical cap formed by slicing $\mathbf{B}$ with hyperplanes at distance $h$ from $\pm a$ respectively, perpendicular to the line joining $a$ and $-a$. Let $A$ be the event that at least one of the two caps $\pm C$ contains no vertex of $V_{n}$. Then

$$
\operatorname{Pr}[A]=2 \operatorname{Pr}\left[C \cap V_{n}=\emptyset\right]=2\left(1-\frac{\operatorname{vol}(C)}{\operatorname{vol}(\mathbf{B})}\right)^{n} \leq 2 \exp \left(-n \frac{\operatorname{vol}(C)}{\operatorname{vol}(\mathbf{B})}\right) .
$$

On the other hand, $\operatorname{vol}(C) / \operatorname{vol}(B)=O\left(h^{(d+1) / 2}\right)$ by (18) of $\S$ A.3, which together with the condition (3) on $h$ implies that $\operatorname{Pr}[A]=o(1)$. That is, $G$ almost always 
Random geometric graph diameter in the unit ball (with Robert B. Ellis and Catherine Yan) Algorithmica 47, no. 4 (2007), $421--438$. The original publication is available at www.springerlink.com: http://dx.doi.org/10.1007/s00453-006-0172-y Open Access version: http://kuscholarworks.ku.edu/dspace/.

contains a vertex in each of $C$ and $-C$. The result now follows from the definition of $G$ and the lower bound (19) on the $\ell_{p}$-distance between $C$ and $-C$.

Note that for all $d \geq 1, h$ can be chosen to satisfy both $(3)$ and $\lim _{n \rightarrow \infty} h / \lambda=0$. Also, if the limit in (3) is a nonnegative constant, then $\lim _{n \rightarrow \infty} \operatorname{Pr}[A]>0$; that is, vertices are not guaranteed in both caps. For the case $p=2$, Proposition 7 can be strengthened by identifying a collection of mutually disjoint antipodal pairs of caps of height $h$ and showing that, almost always, both caps in at least one pair contain a vertex. Such a collection corresponds to an antipodally symmetric spherical code (see $[3])$.

\section{The ABSOlute UPPER BOUND}

In this section we prove that when $G$ is connected, the graph distance $d_{G}(x, y)$ between two vertices $x, y \in V_{n}$ is at most $K\|x-y\|_{p} / \lambda$, where $K>0$ is a constant independent of $n$ and $p$, but dependent on $d$. As a consequence, $\operatorname{diam}(G) \leq$ $K \operatorname{diam}_{p}(\mathbf{B}) / \lambda$. This will not be strong enough to meet (asymptotically) the lower bound in Proposition 7, but does guarantee a short path between any pair of vertices. This fact will be used repeatedly in the proof of the tight upper bound in Theorem 10 of $\S 6$. It is sufficient to prove the following Theorem 8 , since for any two points $x, y \in \mathbb{R}^{d}$, we have $\|x-y\|_{2} \leq d^{1 / 2}\|x-y\|_{p}$.

Theorem 8. Let $d \geq 2$, and suppose that $\lambda=\lambda(n)$ is sufficiently large so that Theorem 6 guarantees that almost always, $G=G_{p}^{d}(\lambda, n)$ is connected. Then for any two points $x, y \in V_{n}$ there exists a constant $K$ independent of $n$ and $p$ such that as $n \rightarrow \infty$, almost always,

$$
d_{G}(x, y) \leq \frac{K\|x-y\|_{2}}{\lambda}
$$

The proof is based on Proposition 9 below. For any two vertices $x, y \in V_{n}$, let

$$
T_{x, y}(k)=\left[\text { convex closure of }\left(B_{2}^{d}(x, k \lambda) \cup B_{2}^{d}(y, k \lambda)\right)\right] \cap \mathbf{B} .
$$

Thus $T_{x, y}$ is a "lozenge"-shaped region. Let $A_{n}(k)$ be the event that there exist two vertices $x, y \in V_{n}$ such that (i) at least one point is inside $B_{2}^{d}(O, 1-(k+\sqrt{d}) \lambda)$, and (ii) there is no path of $G$ that lies in $T_{x, y}(k)$ and connects $x$ and $y$. The proof of our next result uses ingredients from [9, p. 285], adapted and extended for our present purposes.

Proposition 9. Under the same assumptions as in Theorem 8, there exists a constant $k_{0}>0$, such that for all $k>k_{0}$,

$$
\lim _{n \rightarrow \infty} \operatorname{Pr}\left[A_{n}(k)\right]=0 .
$$

Proof. First, we cover the unit ball $\mathbf{B}$ with $d$-dimensional cubes, each of side length $\epsilon \lambda$, where $\epsilon=1 /(4 d)$. Let $L_{d}$ be the set of centers of these cubes, and for each $z \in L_{d}$, denote the closed cube centered at $z$ by $Q_{z}$.

Suppose $A_{n}(k)$ occurs for a pair of vertices $x, y$; without loss of generality, suppose $y \in B_{2}^{d}(O, 1-(k+\sqrt{d}) \lambda)$. Abbreviate $T_{x, y}(k)$ by $T_{x, y}$.

Step 1. First we construct a connected subset $P \subseteq T_{x, y}$ such that

(i) $B_{p}^{d}(P, \lambda / 4) \subseteq \mathbf{B}$;

(ii) $\operatorname{diam}_{2}(P) \geq(k-\sqrt{d}) \lambda$; and

(iii) $B_{p}^{d}(P, \lambda / 4) \cap V_{n}=\emptyset$. 
Random geometric graph diameter in the unit ball (with Robert B. Ellis and Catherine Yan) Algorithmica 47, no. 4 (2007), $421--438$. The original publication is available at www.springerlink.com: http://dx.doi.org/10.1007/s00453-006-0172-y Open Access version: http://kuscholarworks.ku.edu/dspace/.

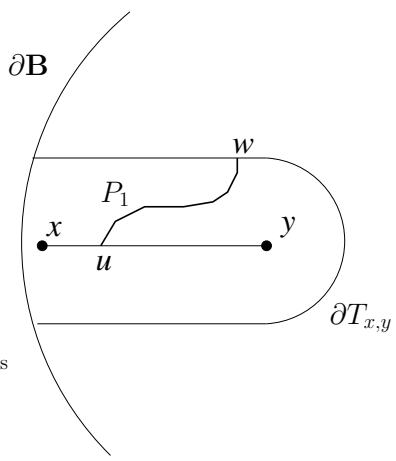

Figure 1. The frontier $P_{1}$ must intersect the line segment between vertices $x, y \in V_{n}$ at some point $u$, and must also satisfy $d_{p}\left(P_{1}, w\right) \leq \lambda / 2$ for some point $w$ on the boundary $\partial T_{x, y} \backslash \partial \mathbf{B}$.

Let $V_{T}=V_{n} \cap T_{x, y}$ be the set of vertices of $G$ lying in $T_{x, y}$. Then $B_{p}^{d}\left(V_{T}, \lambda / 2\right)$ is (topologically) disconnected with $x$ and $y$ lying in different connected components. Let $D_{x}$ be the connected component of $B_{p}^{d}\left(V_{T}, \lambda / 2\right)$ containing $x$. Let $S$ be the closure of the connected component of $T_{x, y} \backslash D_{x}$ containing $y$. Let $T$ be the closure of $T_{x, y} \backslash S$, so that $T$ contains $x$. Then both $S$ and $T$ are connected, and their union is $T_{x, y}$. The lozenge $T_{x, y}$ is simply connected, so it is unicoherent [9, Lemma 9.1]; by definition of unicoherence, since $T_{x, y}$ is the union of closed connected sets $S, T \subseteq$ $T_{x, y}$, then $P_{1}:=S \cap T$ is connected. Since $x \in T, y \in S$, and $P_{1}$ separates $x$ and $y$, any path in $T_{x, y}$ from $x$ to $y$ must pass through $P_{1}$. In particular, $P_{1}$ intersects the line segment joining $x$ and $y$. Let $u$ be one of the intersection points.

Next, we show that there is a point $w$ on $\partial T_{x, y}$ such that $d_{p}\left(P_{1}, w\right) \leq \lambda / 2$, and derive from this that $\operatorname{diam}_{2}\left(P_{1}\right) \geq(k-\sqrt{d} / 2) \lambda$ provided that the $\ell_{2}$-distance between $u$ and $w$ is at least $k \lambda$ (see Figure 1 for an illustration). To achieve this, we must avoid the case that $w$ lies in the boundary of $\mathbf{B}$. To this end, let $C_{1}=\partial \mathbf{B} \cap T_{x, y}$, and let $C_{2}=\partial T_{x, y} \backslash C_{1}$. If $d_{p}\left(P_{1}, C_{2}\right) \geq \lambda / 2$, then $C_{2}$ must be a subset either of $S$ or of $T$; without loss of generality, assume $S$. Then $y$ is disconnected from $x$ in $G$, which happens with probability tending to zero by Theorem 6. Hence, almost always, $d_{p}\left(P_{1}, C_{2}\right)<\lambda / 2$. It follows that there is a point $w \in C_{2}$ such that $d_{p}\left(P_{1}, w\right) \leq \lambda / 2$. Furthermore, $B_{p}^{d}\left(P_{1}, \lambda / 2\right) \cap V_{n}=\emptyset$, by definition of $P_{1}$ as the intersection of $S$ and $T$.

As constructed, $P_{1}$ may be too close to the boundary of $\mathbf{B}$ so that some cube $Q_{z}$ intersecting $P_{1}$ might not lie entirely inside $\mathbf{B}$. To overcome this, we let $P$ be obtained from $P_{1}$ by moving every point toward $O$ by $\lambda / 4$ under the transformation $x \rightarrow x-(\lambda / 4)\left(x /\|x\|_{2}\right)$. Then $P$ is connected, and $\operatorname{diam}_{2}(P) \geq \operatorname{diam}_{2}\left(P_{1}\right)-\lambda / 2 \geq$ $(k-\sqrt{d}) \lambda$; that is $P$ satisfies conditions (i-iii).

Step 2. We now show that when $k$ is large enough, the probability

$$
\operatorname{Pr}\left[Q_{z} \cap V_{n}=\emptyset \text { for every } Q_{z} \subseteq B_{p}^{d}(P, \lambda / 4)\right]
$$

tends to zero. Let $\omega$ be the set of points $z \in L_{d}$ such that $Q_{z} \cap P \neq \emptyset$. Since $P$ is connected, $\omega$ is a $*$-connected subset of $L_{d}$; that is, the union of the corresponding 
Random geometric graph diameter in the unit ball (with Robert B. Ellis and Catherine Yan) Algorithmica 47, no. 4 (2007), $421--438$. The original publication is available at www.springerlink.com: http://dx.doi.org/10.1007/s00453-006-0172-y Open Access version: http://kuscholarworks.ku.edu/dspace/.

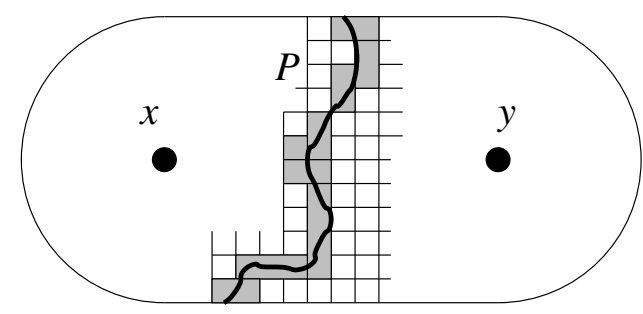

Figure 2. Two vertices $x, y \in V_{n}$ which are not connected by any path in $T_{x, y}(k)$, and the "frontier" $P$ separating them, when $d=2$. The gray squares are the $*$-connected subset $\omega$ intersecting $P$ of the set of squares covering $\mathbf{B}$.

set of cubes is (topologically) connected (see Figure 2). For each $z \in \omega$, we have $Q_{z} \cap P \neq \emptyset$ and $\epsilon \leq 1 /\left(4 d^{1 / p}\right)$; hence $Q_{z} \subseteq B_{p}^{d}(P, \lambda / 4)$. By considering the $\ell_{2^{-}}$ diameter of $P$, we see that $\omega$ contains at least $4 \sqrt{d}(k-\sqrt{d})$ points. Hence we have a $*$-connected subset $\omega \subseteq L_{d}$ with cardinality at least $4 \sqrt{d}(k-\sqrt{d})$ such that $Q_{z} \cap V_{n}=\emptyset$ for all $z \in \omega$. We show that the probability of such an event is $o(1)$.

Let $\mathcal{C}_{d, i}$ denote the collection of $*$-connected sets of $\omega \subseteq L_{d}$ of cardinality $i$. It is known that the number of $*$-connected subsets of $\mathbb{Z}^{d}$ of cardinality $i$ containing the origin is at most $2^{3^{d} i}$ (see, for example, [9, Lemma 9.3]). Since $\left|L_{d}\right| \leq(2 /(\epsilon \lambda))^{d}$, we have $\mathcal{C}_{d, i} \leq(2 /(\epsilon \lambda))^{d} 2^{3^{d} i} \leq 2^{d}(\epsilon \lambda)^{-d} e^{3^{d} i}$. Therefore

$$
\begin{aligned}
\operatorname{Pr}\left[A_{n}(k)\right] & \leq \sum_{i \geq 4 \sqrt{d}(k-\sqrt{d}))} \sum_{\omega \in \mathcal{C}_{d, i}} \operatorname{Pr}\left[V_{n} \cap\left(\cup_{z \in \omega} Q_{z}\right)=\emptyset\right] \\
& \leq \sum_{i \geq 4 \sqrt{d}(k-\sqrt{d}))} 2^{d}(\epsilon \lambda)^{-d} \exp \left(3^{d} i\right)\left(1-\frac{i}{\operatorname{vol}(\mathbf{B})}(\epsilon \lambda)^{d}\right)^{n} \\
& \leq \sum_{i \geq 4 \sqrt{d}(k-\sqrt{d}))} c n \exp \left(-i \epsilon^{d}(d-1) \ln n /(d \alpha \operatorname{vol}(\mathbf{B}))\right) \\
& =O\left(n^{1-(4 \sqrt{d}(k-\sqrt{d})(d-1)) /\left(d \alpha \operatorname{vol}(\mathbf{B})(4 d)^{d}\right)}\right),
\end{aligned}
$$

where $c$ is a constant and $\alpha=\alpha_{p}^{d}$ is the constant of (1). To justify inequality (4), when $n$ is sufficiently large, we have $3^{d}<\epsilon^{d}(d-1) \ln n /(d \alpha \operatorname{vol}(\mathbf{B}))$. The order bound in (5) is immediate by geometric series, and the resulting quantity is $o(1)$ provided $k>\sqrt{d}+d \alpha \operatorname{vol}(\mathbf{B})(4 d)^{d} /(4 \sqrt{d}(d-1))$, which proves the existence of $k_{0}$ in the proposition.

Proof of Theorem 8. Fix $k>k_{0}$ as in Proposition 9. Let $x$ and $y$ be two vertices in $V_{n}$ with $\|x-y\|_{p}>\lambda$. If at least one of $x, y$ lies in $B_{2}^{d}(O, 1-(k+\sqrt{d}) \lambda)$, then, almost always, there is a path of $G$ connecting $x$ and $y$ in $T_{x, y}$. Suppose the shortest path between $x$ and $y$ in $T_{x, y}$ has length $g$. Then the $\ell_{p}$-balls of radius $\lambda / 2$ around every other vertex in the path must be pairwise disjoint, and each must lie inside the convex closure of $B_{2}^{d}(x,(k+\sqrt{d} / 2) \lambda) \cup B_{2}^{d}(y,(k+\sqrt{d} / 2) \lambda)$. By comparing the 
Random geometric graph diameter in the unit ball (with Robert B. Ellis and Catherine Yan) Algorithmica 47, no. 4 (2007), $421--438$. The original publication is available at www.springerlink.com: http://dx.doi.org/10.1007/s00453-006-0172-y Open Access version: http://kuscholarworks.ku.edu/dspace/.

volume of the $\ell_{p}$-balls of radius $\lambda / 2$ to the volume of $T_{x, y}$, we obtain

$$
\begin{aligned}
\left\lfloor\frac{g}{2}\right\rfloor \operatorname{vol}\left(B_{p}^{d}(x, \lambda / 2)\right) \leq & \operatorname{vol}\left(B_{2}^{d}(x,(k+\sqrt{d} / 2) \lambda)\right) \\
& +d_{2}(x, y) \cdot \operatorname{vol}\left(B_{2}^{d-1}(x,(k+\sqrt{d} / 2) \lambda)\right),
\end{aligned}
$$

which implies that $g \leq K_{1}+K_{2} d_{2}(x, y) / \lambda \leq\left(K_{1} \sqrt{d}+K_{2}\right) d_{2}(x, y) / \lambda$, where $K_{1}$ and $K_{2}$ are constants independent of $n$ and $p$.

If both $x$ and $y$ lie outside $B_{2}^{d}(O, 1-(k+\sqrt{d}) \lambda)$, then we can travel from $x$ to an intermediate vertex $x_{1}$ just inside $B(O, 1-(k+\sqrt{d}) \lambda)$ via a path of bounded length, and then on to $y$. To this end, let $r=\max \left\{\left(\alpha_{p}^{d}\right)^{1 / d} \sqrt{d}, \sqrt{d}\right\}$, and let $E_{n}(k)$ be the event that there is a vertex $z \in V_{n}$ such that $z \notin B(O, 1-(k+\sqrt{d}) \lambda)$ and $V_{n} \cap(B(O, 1-(k+\sqrt{d}) \lambda) \cap B(z,(k+\sqrt{d}+2 r) \lambda))=\emptyset$. Then

$$
\operatorname{Pr}\left[E_{n}(k)\right] \leq n\left(1-(1-(k+\sqrt{d}) \lambda)^{d}\right)\left(1-(\sqrt{d} \lambda)^{d}\right)^{n}=o(1) .
$$

Applying this observation with $z=x$, we can find a point $x_{1} \in V_{n}$ inside $B(O, 1-$ $(k+\sqrt{d}) \lambda) \cap B(x,(k+\sqrt{d}+2 r) \lambda)$. By the preceding argument we can first travel from $x$ to $x_{1}$ in $K d_{2}\left(x, x_{1}\right) / \lambda$ steps, and then from $x_{1}$ to $y$ in $K d_{2}\left(x_{1}, y\right) / \lambda$ steps. The total length of the path is no more than $K\left(d_{2}(x, y)+2 d_{2}\left(x, x_{1}\right)\right) / \lambda$. Theorem 8 follows from the fact that $d_{2}\left(x, x_{1}\right) \leq(k+\sqrt{d}+2 r) \lambda$.

We briefly discuss the case that $d=1$, so that $\mathbf{B}$ is the interval $[-1,1] \subset \mathbb{R}$. Suppose that $\lambda=\lambda(n)$ is sufficiently large so that Theorem 6 guarantees that, almost always, $G_{p}^{1}(\lambda, n)$ is connected. For any two vertices $x, y$, the shortest path between them clearly consists of a strictly increasing set of vertices $x=x_{0}<x_{1}<$ $x_{2}<\cdots<x_{d_{G}(x, y)}=y$. Moreover, the balls $B\left(x_{0}, \lambda / 2\right), B\left(x_{2}, \lambda / 2\right), B\left(x_{4}, \lambda / 2\right), \ldots$ must be pairwise disjoint (else some $x_{i}$ is redundant). Hence $|x-y| \geq\left\lceil d_{G}(x, y) / 2\right\rceil \lambda$, and from this it is not hard to deduce that $d_{G}(x, y) \leq 2|x-y| / \lambda$.

\section{The ASYMPtotically tight UPPER BOUND}

In this section, we improve the upper bound in Theorem 8 , reducing the constant $K$ to $\operatorname{diam}_{p}(\mathbf{B})$ (asymptotically). Our main result is as follows:

Theorem 10. Let $d \geq 2$ and $p \in[1, \infty]$, and suppose that $\lambda=\lambda(n)$ is sufficiently large so that Theorem 6 guarantees that almost always, $G=G_{p}^{d}(\lambda, n)$ is connected. Then as $n \rightarrow \infty$, almost always,

$$
\operatorname{diam}(G) \leq \begin{cases}\left(2 d^{1 / p-1 / 2}+O\left((\ln \ln n / \ln n)^{1 / d}\right)\right) / \lambda & \text { when } 1 \leq p \leq 2, \\ \left(2+O\left((\ln \ln n / \ln n)^{1 / d}\right)\right) / \lambda & \text { when } 2 \leq p \leq \infty\end{cases}
$$

That is, almost always, $\operatorname{diam}(G) \leq \operatorname{diam}_{p}(\mathbf{B})\left(1+O\left((\ln \ln n / \ln n)^{1 / d}\right)\right) / \lambda$.

The proof uses the geometric ingredients of pins and pincushions. A pin consists of a collection of evenly spaced, overlapping $\ell_{p}$-balls whose centers lie on a diameter of the Euclidean unit $d$-ball B. By making suitable choices for the geometry, we can ensure that each intersection of consecutive balls contains a vertex in $V_{n}$, so that the pin provides a "highway" through $G$. Having done this, we construct a pincushion so that every point of $\mathbf{B}$ is reasonably close to an $\ell_{p}$-ball in one of its constituent pins. The following definitions are illustrated in Figure 3. 
Random geometric graph diameter in the unit ball (with Robert B. Ellis and Catherine Yan) Algorithmica 47, no. 4 (2007), $421--438$. The original publication is available at www.springerlink.com: http://dx.doi.org/10.1007/s00453-006-0172-y Open Access version: http://kuscholarworks.ku.edu/dspace/.
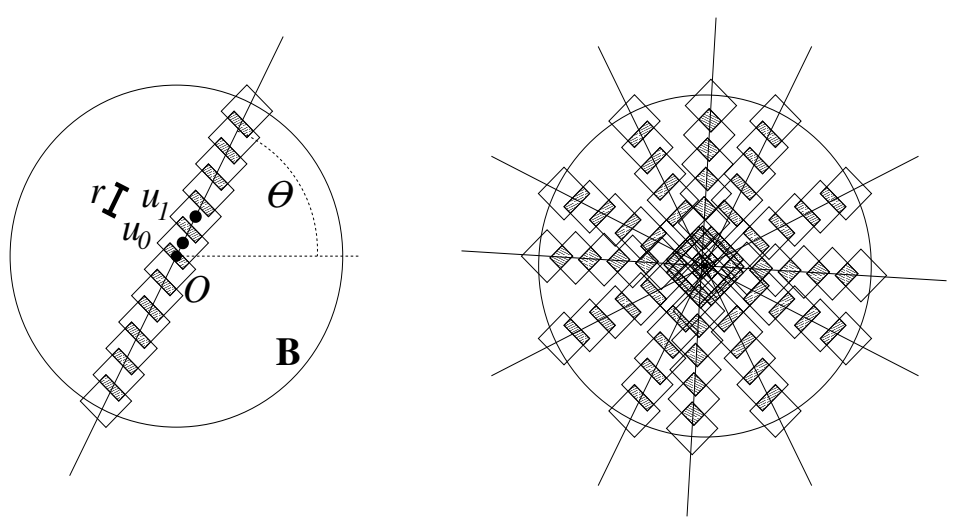

Figure 3. (a) A pin in the unit circle in $\mathbb{R}^{2}$, with angle $\theta$. Here $p=1$, so that the $\ell_{p}$-balls are diamonds, and $r$ is the $\ell_{2}$-distance between consecutive centers (such as $u_{0}$ and $u_{1}$ ) of $\ell_{1}$-balls. The petals are the shaded regions. (b) A pincushion consisting of several pins.

Definition 11 (Pins). Fix $d \geq 2, p \in[1, \infty], \theta \in(-\pi / 2, \pi / 2], \underline{\phi}=\left(\phi_{3}, \ldots, \phi_{d}\right) \in$ $[0, \pi / 2]^{d}$, and $\lambda, r>0$. For $m \in \mathbb{Z}$, put

$$
u_{m}=u_{m}(r, \theta)=\left(\frac{r}{2}+r m\right) \cdot\left(x_{1}, \ldots, x_{d}\right) \in \mathbb{R}^{d},
$$

where

$$
x_{1}=\cos \theta \prod_{i=3}^{d} \sin \phi_{i}, \quad x_{2}=\sin \theta \prod_{i=3}^{d} \sin \phi_{i}, \quad x_{j}=\cos \phi_{j} \prod_{i=j+1}^{d} \sin \phi_{i} \text { for } 3 \leq j \leq d .
$$

The corresponding pin $U(d, p, \theta, \underline{\phi}, r, \lambda)$ consists of the points $\left\{u_{m}: m \in \mathbb{Z}\right\} \cap \mathbf{B}$, together with a collection of $\ell_{p}$-balls of radius $\lambda / 2$, one centered at each point $u_{m}$. Note that the total number of $\ell_{p}$-balls is $1+2\left\lfloor\frac{1}{r}\right\rfloor$.

Definition 12 (Pincushions). Fix $d \geq 2,1 \leq p \leq \infty$, and $\sigma \in \mathbb{Z}^{+}$. The corresponding pincushion (with parameters $d, p, \sigma, r, \lambda)$ is the set of $(2 \sigma)^{d-1}$ pins

$$
\mathcal{U}:=\left\{U(d, p, \theta, \underline{\phi}, r, \lambda): \theta, \phi_{i} \in\left\{0, \frac{\pi}{2 \sigma}, \frac{2 \pi}{2 \sigma}, \ldots, \frac{(2 \sigma-1) \pi}{2 \sigma}\right\}\right\} .
$$

Definition 13 (Petals). Let $U$ be a pin. A petal is the region of intersection of two overlapping $\ell_{p}$-balls on $U$ (the shaded regions in Figure 3 ). A petal is nonempty if it contains a vertex of $V_{n}$.

The probability that a petal is nonempty depends on its volume, which depends in turn on the parameters of the corresponding pin. Certainly, we must choose $r$ so that the petal has positive volume: for example, it suffices to take $r \leq\left(\operatorname{diam}_{2}(\mathbf{B}) / \operatorname{diam}_{p}(\mathbf{B})\right) \lambda$. Unfortunately, the volume is difficult to calculate exactly. Even finding the minimum volume over all angles, that is,

$$
\xi=\xi_{p}^{d}(r, \lambda / 2):=\inf \left\{\operatorname{vol}\left(B_{p}^{d}(x, \lambda / 2) \cap B_{p}^{d}(y, \lambda / 2)\right): x, y \in \mathbf{B},\|x-y\|_{2}=r\right\} .
$$


Random geometric graph diameter in the unit ball (with Robert B. Ellis and Catherine Yan) Algorithmica 47, no. 4 (2007), $421--438$. The original publication is available at www.springerlink.com: http://dx.doi.org/10.1007/s00453-006-0172-y Open Access version: http://kuscholarworks.ku.edu/dspace/.

requires integrals that are not easily evaluated (although for fixed $d$ and $r$, it is certainly true that $\xi=\Theta\left(\lambda^{d}\right)$ ). The easiest way to find a lower bound for $\xi$ is to inscribe another $\ell_{p}$-ball in the petal.

Lemma 14. Let $d \geq 1, p \in[1, \infty], \lambda>0$ and $0 \leq r \leq\left(\operatorname{diam}_{p}(\mathbf{B}) / \operatorname{diam}_{2}(\mathbf{B})\right) \lambda$. Then, for all $x, y \in \mathbf{B}$ with $\|x-y\|_{2}=r$,

$$
B_{2}^{d}\left(\frac{x+y}{2}, r^{\prime}\right) \subseteq B_{p}^{d}(x, \lambda / 2) \cap B_{p}^{d}(y, \lambda / 2),
$$

where

$$
r^{\prime}= \begin{cases}\frac{\lambda}{2} d^{1 / 2-1 / p}-\frac{r}{2} & \text { when } 1 \leq p \leq 2 \\ \frac{\lambda}{2}-\frac{r}{2} & \text { when } 2 \leq p \leq \infty\end{cases}
$$

In particular,

$$
\frac{\xi}{\operatorname{vol}(\mathbf{B})} \geq \begin{cases}\left(\frac{\lambda}{2} d^{1 / 2-1 / p}-\frac{r}{2}\right)^{d} & \text { when } 1 \leq p \leq 2 \\ \left(\frac{\lambda}{2}-\frac{r}{2}\right)^{d} & \text { when } 2 \leq p \leq \infty\end{cases}
$$

Proof. Let $z \in B_{2}^{d}\left(\frac{x+y}{2}, r^{\prime}\right)$. By the triangle inequality,

$$
\|z-x\|_{2} \leq\left\|z-\frac{x+y}{2}\right\|_{2}+\left\|\frac{x+y}{2}-x\right\|_{2} \leq r^{\prime}+\frac{r}{2},
$$

which, together with (17), implies that $\|z-x\|_{p} \leq \lambda / 2$. That is, $z \in B_{p}^{d}(x, \lambda / 2)$. The same argument implies that $z \in B_{p}^{d}(y, \lambda / 2)$. The bound on $\xi$ is then a simple application of (14).

For the remainder of this section, we work with the pincushion $\mathcal{U}$ defined by

$$
\sigma=\sigma(n)=\left\lfloor(\ln n)^{1 / d}\right\rfloor
$$

and

$$
r=r(n)= \begin{cases}\lambda d^{1 / 2-1 / p}(1-\rho(n)) & \text { when } 1 \leq p \leq 2, \\ \lambda(1-\rho(n)) & \text { when } 2 \leq p \leq \infty\end{cases}
$$

where

$$
\rho=\rho(n)= \begin{cases}2 d^{1 / p-1 / 2}(\ln \ln n / \ln n)^{1 / d} & \text { when } 1 \leq p \leq 2, \\ 2(\ln \ln n / \ln n)^{1 / d} & \text { when } 2 \leq p \leq \infty .\end{cases}
$$

Let $\tau_{U}=\tau_{U}(n)$ be the number of empty petals along the pin $U \in \mathcal{U}$, and define

$$
\tau=\tau(n)=\max \left\{\tau_{U}(n): U \in \mathcal{U}\right\} .
$$

We first calculate an upper bound on $\tau$.

Lemma 15. With the assumptions of Theorem 10, and the parameters $\sigma, r, \rho$ as just defined, almost always,

$$
\tau \leq \sigma^{(d-1) / 2} \frac{2}{r} \exp \left(\frac{-n \xi}{\operatorname{vol}(\mathbf{B})}\right)
$$

Proof. Denote the right-hand side of the desired inequality by $T$. By linearity of expectation,

$$
\begin{aligned}
\operatorname{Pr}[\tau \geq T] & \leq \mathbb{E}\left[\left|\left\{U: \tau_{U} \geq T\right\}\right|\right] \\
& \leq(2 \sigma)^{d-1} \cdot \operatorname{Pr}\left[\tau_{U^{*}} \geq T\right]
\end{aligned}
$$


Random geometric graph diameter in the unit ball (with Robert B. Ellis and Catherine Yan) Algorithmica 47, no. 4 (2007), $421--438$. The original publication is available at www.springerlink.com: http://dx.doi.org/10.1007/s00453-006-0172-y Open Access version: http://kuscholarworks.ku.edu/dspace/.

where $U^{*}$ is chosen so as to maximize $\operatorname{Pr}\left[\tau_{U} \geq T\right]$. Let $X_{i}$ be the indicator random variable of the event that the $i$ th petal of $U^{*}$ is empty, and let $X=\sum_{i} X_{i}$. Now $U^{*}$ contains at most $2 / r$ petals, so by linearity of expectation,

$$
\mathbb{E}[X] \leq \frac{2}{r}\left(1-\frac{\xi}{\operatorname{vol}(\mathbf{B})}\right)^{n} \leq \frac{2}{r} \exp \left(\frac{-n \xi}{\operatorname{vol}(\mathbf{B})}\right)
$$

Also,

$$
\operatorname{var}[X] \leq \mathbb{E}[X]+\sum_{i \neq j} \operatorname{cov}\left(X_{i}, X_{j}\right)
$$

where the covariance $\operatorname{cov}\left(X_{i}, X_{j}\right)$ for $i \neq j$ is

$$
\begin{aligned}
\operatorname{cov}\left(X_{i}, X_{j}\right) & =\operatorname{Pr}\left[X_{i} X_{j}=1\right]-\operatorname{Pr}\left[X_{i}=1\right] \cdot \operatorname{Pr}\left[X_{j}=1\right] \\
& =\left(1-\frac{2 \xi}{\operatorname{vol}(\mathbf{B})}\right)^{n}-\left(1-\frac{\xi}{\operatorname{vol}(\mathbf{B})}\right)^{2 n} \\
& \leq o(1) \cdot \exp \left(\frac{-2 n \xi}{\operatorname{vol}(\mathbf{B})}\right) .
\end{aligned}
$$

Combining (8), (9) and (10) gives

$$
\operatorname{var}[X] \leq \frac{2}{r} \exp \left(\frac{-n \xi}{\operatorname{vol}(\mathbf{B})}\right)+\frac{4}{r^{2}} o(1) \cdot \exp \left(\frac{-2 n \xi}{\operatorname{vol}(\mathbf{B})}\right)
$$

By Chebyshev's inequality (cf. [1]) and the bounds for $\mathbb{E}[X]$ and $\operatorname{var}[X]$ in $(8)$ and (11),

$$
\begin{aligned}
\operatorname{Pr}[X \geq T] & \leq \operatorname{Pr}[|X-\mathbb{E}[X]| \geq T-\mathbb{E}[X]] \\
& \leq \frac{\operatorname{var}[X]}{(T-\mathbb{E}[X])^{2}}=o\left(\frac{1}{\sigma^{d-1}}\right) .
\end{aligned}
$$

Now $\operatorname{Pr}\left[\tau_{U} \geq T\right]=\operatorname{Pr}[X \geq T]$. Therefore, substituting this last bound into (7) gives $\operatorname{Pr}[\tau \geq T]=o(1)$, which implies the desired result.

We can now prove the main result of this section.

Proof of Theorem 10. Let $x, y \in V_{n}$. We will find vertices $x_{1}$ and $y_{1}$ near $x$ and $y$ respectively, belonging to petals of the pincushion $\mathcal{U}$. From each of $x_{1}, y_{1}$, we walk along the appropriate pin to points $x_{2}, y_{2}$ near the origin and belonging to petals on the same pin as $x_{1}$ and $y_{1}$, respectively. We will then use Theorem 8 to construct a path from $x_{2}$ to $y_{2}$, as well as any "detours" needed in case there are missing edges in the paths along the pins. Without loss of generality, we may assume that

$$
\|x\|_{2},\|y\|_{2} \geq(\tau+3 / 2) r .
$$

The justification for this is deferred until the end of the proof.

Let $R_{x}$ be the (possibly empty) petal nearest to $x$, and let $U_{x}$ be the pin containing $R_{x}$. When $n$ is sufficiently large, the distance from $x$ to $R_{x}$ is at most $d \pi / 2 \sigma$. By definition of $\tau$, there is another vertex $x_{1} \in V_{n}$, which also lies in a petal on $U_{x}$, but is closer to the origin, so that $\left\|x-x_{1}\right\|_{2} \leq d \pi / 2 \sigma+r(\tau+1)$. Repeat these constructions for $y$ to obtain an analogous vertex $y_{1}$. Then

$$
\left\|x-x_{1}\right\|_{2},\left\|y-y_{1}\right\|_{2} \leq \frac{d \pi}{2 \sigma}+r(\tau+1),
$$


Random geometric graph diameter in the unit ball (with Robert B. Ellis and Catherine Yan) Algorithmica 47, no. 4 (2007), $421--438$. The original publication is available at www.springerlink.com: http://dx.doi.org/10.1007/s00453-006-0172-y Open Access version: http://kuscholarworks.ku.edu/dspace/.

and, by Theorem 8 ,

$$
d_{G}\left(x, x_{1}\right), d_{G}\left(y, y_{1}\right) \leq\left(\frac{d \pi}{2 \sigma}+r(\tau+1)\right) \frac{K}{\lambda} .
$$

By definition of $\tau$, there is a vertex $x_{2}$ that belongs to a petal $R_{x_{2}}$ on $U_{x}$ (indeed, lying on the same side of $O$ along $\left.U_{x}\right)$ with $R_{x_{2}} \subseteq B_{2}^{d}(O, r(\tau+3 / 2))$. In the worst case, all empty petals in $U_{x}$ occur non-consecutively between $x_{1}$ and $x_{2}$, so for $n$ large enough, we have

$$
\begin{aligned}
d_{G}\left(x_{1}, x_{2}\right) & \leq \frac{\left\|x_{1}\right\|_{2}}{r}+\frac{2 K r \tau}{\lambda} \\
& \leq \frac{\|x\|_{2}}{r}+\frac{d \pi}{2 \sigma r}+\frac{2 K r \tau}{\lambda}
\end{aligned}
$$

The same construction goes through if we replace the $x$ 's with $y$ 's. Moreover, $\left\|x_{2}-y_{2}\right\|_{2} \leq(2 \tau+3) r$, so the shortest path in $G$ between $x_{2}$ and $y_{2}$ satisfies

$$
d_{G}\left(x_{2}, y_{2}\right) \leq \frac{(2 \tau+3) r K}{\lambda} .
$$

Concatenating all the above paths, we find that

$$
\begin{aligned}
d_{G}(x, y) & \leq d_{G}\left(x, x_{1}\right)+d_{G}\left(x_{1}, x_{2}\right)+d_{G}\left(x_{2}, y_{2}\right)+d_{G}\left(y_{2}, y_{1}\right)+d_{G}\left(y_{1}, y\right) \\
& \leq \frac{\|x\|_{2}+\|y\|_{2}}{r}+\left(\frac{d \pi}{\sigma}+(8 \tau+5) r\right) \frac{K}{\lambda}+\frac{d \pi}{\sigma r} \\
& \leq \frac{\operatorname{diam}_{p}(\mathbf{B})}{\operatorname{diam}_{2}(\mathbf{B})} \frac{\|x\|_{2}+\|y\|_{2}+O(\rho)}{\lambda}+O(\tau)+O\left(\frac{(\ln n)^{-1 / d}}{\lambda}\right) .
\end{aligned}
$$

By the definitions of $r$ and $\rho$ given in (6b) and (6c), and the bounds on $\xi$ and $\tau$ (Lemmas 14 and 15), it follows that

$$
\frac{\xi}{\operatorname{vol}(\mathbf{B})} \geq \lambda^{d} \frac{\ln \ln n}{\ln n} \geq \frac{\ln \ln n}{n},
$$

where the second inequality follows from the assumption that $\lambda$ is above the threshold for connectivity in Theorem 6 . Therefore

$$
\begin{aligned}
\tau & \leq \frac{2 \sigma^{(d-1) / 2}}{r} \exp \left(\frac{-n \xi}{\operatorname{vol}(\mathbf{B})}\right) \\
& \leq \frac{4}{\lambda}(\ln n)^{(d-1) /(2 d)}(\ln n)^{-1}=\frac{O\left((\ln n)^{-(d+1) /(2 d)}\right)}{\lambda}=\frac{o(\rho)}{\lambda} .
\end{aligned}
$$

Plugging this bound for $\tau$ into (13) gives

$$
d_{G}(x, y) \leq \frac{\operatorname{diam}_{p}(\mathbf{B})}{\operatorname{diam}_{2}(\mathbf{B})} \frac{\|x\|_{2}+\|y\|_{2}+O(\rho)}{\lambda},
$$

and the theorem follows.

We now explain the assumption (12). If $\|x\|_{2},\|y\|_{2} \leq(\tau+3 / 2) r$, then by Theorem $8, d_{G}(u, v) \leq(2 \tau+3) r K / \lambda=o(\rho) / \lambda$. On the other hand, if $\|x\|_{2} \leq$ $(\tau+3 / 2) r \leq\|y\|_{2}$, then, almost always, there is a vertex $x_{2} \in B_{2}^{d}(O,(\tau+3 / 2) r)$ belonging to some petal. By the preceding argument, $d_{G}(x, y) \leq o(\rho / \lambda)+d_{G}\left(x_{2}, y\right) \leq$ $\left(\operatorname{diam}_{p}(\mathbf{B}) / \operatorname{diam}_{2}(\mathbf{B})\right)\left(\|y\|_{2}+O(\rho)\right) / \lambda$. 
Random geometric graph diameter in the unit ball (with Robert B. Ellis and Catherine Yan) Algorithmica 47, no. 4 (2007), $421--438$.

The original publication is available at www.springerlink.com: http://dx.doi.org/10.1007/s00453-006-0172-y Open Access version: http://kuscholarworks.ku.edu/dspace/.

We conclude with two remarks. First, the proof of Theorem 10 can easily be adapted to the case $d=1$ to obtain the upper $\operatorname{bound} \operatorname{diam}\left(G_{p}^{1}(\lambda, n)\right) \leq$ $(2+O(\ln \ln n / \ln n)) / \lambda$. (Note that when $d=1$ a pincushion consists of just one pin.) Second, the technique of Theorem 10 can be extended to obtain the stronger result $d_{G}(x, y) \leq\left(\|x-y\|_{p}+O\left((\ln \ln n / \ln n)^{1 / d}\right)\right) / \lambda$, so that the graph distance approximates the $\ell_{p}$-metric. Each pin is replaced by $\sigma^{d-1}$ evenly spaced parallel pins. We can still bound $\tau$ by $o(\rho) / \lambda$, but now any two vertices $x$ and $y$ are close to the same pin, on which a short path from $x$ to $y$ is found. We refer the reader to [6] for details.

\section{ACKNOWLEDGEMENTS}

The authors thank Thomas Schlumprecht, Joel Spencer and Dennis Stanton for various helpful discussions and comments. We also thank the anonymous referees for several helpful suggestions.

\section{Appendix A. Facts about $\ell_{p^{-}}$And Spherical geOmetry}

In the body of the article, we used various facts about the Euclidean and $\ell_{p^{-}}$ geometry of balls and spherical caps. None of these facts are difficult; however, for convenience we present them together here along with brief proofs.

A.1. Volume of the $\ell_{p}$-unit ball. Fix $p \in[1, \infty]$ and an integer $d \geq 1$. Let $B_{p}^{d}(r)$ be the $\ell_{p}$-ball centered at the origin in $\mathbb{R}^{d}$ with radius $r$ :

$$
B_{p}^{d}(r)=\left\{x \in \mathbb{R}^{d}: \sum_{i=1}^{d}\left|x_{i}\right|^{p} \leq r^{p}\right\} .
$$

We will show that the (d-dimensional) volume of $B_{p}^{d}(r)$ is

$$
\operatorname{vol}\left(B_{p}^{d}(r)\right)=\frac{(2 r)^{d} \Gamma\left(\frac{p+1}{p}\right)^{d}}{\Gamma\left(\frac{p+d}{p}\right)},
$$

where $\Gamma$ is the usual gamma function [11]. For $d=1$ this is trivial. For $d>1$ we have

$$
\operatorname{vol}\left(B_{p}^{d}(r)\right)=2 \int_{0}^{r} \operatorname{vol}\left(B_{p}^{d-1}\left(\left(r^{p}-x^{p}\right)^{1 / p}\right)\right) d x
$$

Make the substitution $u=x^{p} / r^{p}, x=r u^{1 / p}, d x=(r / p) u^{(1-p) / p} d u$. By induction on $d$, we obtain

$$
\operatorname{vol}\left(B_{p}^{d}(r)\right)=\frac{2^{d} r^{d} \Gamma\left(\frac{p+1}{p}\right)^{d-1}}{p \Gamma\left(\frac{p+d-1}{p}\right)} \int_{0}^{1}(1-u)^{\frac{d-1}{p}} u^{\frac{1-p}{p}} d u
$$

Evaluating this integral as in $[11, \S 12.4]$ yields the desired formula (14). It follows that

$$
\alpha_{p}^{d}:=\frac{\operatorname{vol}\left(B_{p}^{d}(r)\right)}{\operatorname{vol}\left(B_{2}^{d}(r)\right)}=\frac{\Gamma\left(\frac{p+1}{p}\right)^{d} \cdot \Gamma\left(\frac{2+d}{2}\right)}{\Gamma\left(\frac{3}{2}\right)^{d} \cdot \Gamma\left(\frac{p+d}{p}\right)} .
$$


Random geometric graph diameter in the unit ball (with Robert B. Ellis and Catherine Yan) Algorithmica 47, no. 4 (2007), $421--438$. The original publication is available at www.springerlink.com: http://dx.doi.org/10.1007/s00453-006-0172-y Open Access version: http://kuscholarworks.ku.edu/dspace/.

A.2. $\ell_{p}$-antipodes on the Euclidean sphere. Let $\mathbf{B}=B_{2}^{d}(1)$ be the Euclidean unit ball, centered at the origin in $\mathbb{R}^{d}$, and let $p \neq 2$. We wish to calculate the $\ell_{p}$-diameter of $\mathbf{B}$, that is,

$$
\operatorname{diam}_{p}(\mathbf{B})=\max \left\{\|x-y\|_{p}: x, y \in \mathbf{B}\right\} .
$$

A pair of points of $\mathbf{B}$ at distance $\operatorname{diam}_{p}(\mathbf{B})$ are called $\ell_{p}$-antipodes. If $d=1$, then $\mathbf{B}$ is a line segment and the only antipodes are its endpoints. Of course, if $p=2$, then the antipodes are the pairs $\pm x$ with $\|x\|_{2}=1$.

Suppose that $d>1$. Every pair of antipodes $x, y$ must satisfy $\|x\|_{2}=\|y\|_{2}=1$. Without loss of generality, we may assume $x_{i} \geq 0 \geq y_{i}$ for every $i$. Using the method of Lagrange multipliers (with objective function $\left.\left(\|x-y\|_{p}\right)^{p}=\sum_{i=1}^{d}\left(x_{i}-y_{i}\right)^{p}\right)$, we find that for every $i$,

$$
p\left(x_{i}-y_{i}\right)^{p-1}=2 \lambda x_{i}=-2 \mu y_{i},
$$

where $\lambda$ and $\mu$ are nonzero constants. In particular $y_{i}=(-\lambda / \mu) x_{i}$ for every $i$, so $y=-x$. (That is, every pair of $\ell_{p}$-antipodes on $\mathbf{B}$ is a pair of $\ell_{2}$-antipodes.) Substituting for $y_{i}$ in (16) gives

$$
\nu x_{i}^{p-1}=x_{i},
$$

where $\nu$ is some nonzero constant. In particular, the set of coordinates $\left\{x_{1}, \ldots, x_{d}\right\}$ can contain at most one nonzero value, so either $x$ is a coordinate unit vector or else $x_{i}=d^{-1 / 2}$ for every $i$. In the first case $\|(-x)-x\|_{p}=2$, while in the second case $\|(-x)-x\|_{p}=2 d^{-1 / 2+1 / p}$. In summary:

Proposition 16. Let $d \geq 2$, and let $\mathbf{B}=B_{2}^{d}(1)$ be the Euclidean unit ball, centered at the origin in $\mathbb{R}^{d}$. Then the pairs of $\ell_{p}$-antipodes on $\mathbf{B}$ are precisely the pairs $\{ \pm x\}$ satisfying the following additional conditions:

$$
\begin{cases}\left|x_{1}\right|=\cdots=\left|x_{d}\right|=d^{-1 / 2} & \text { when } 1 \leq p<2, \\ \|x\|_{p}=1 & \text { when } p=2, \\ x \text { is a coordinate unit vector } & \text { when } 2<p \leq \infty .\end{cases}
$$

In particular, the $\ell_{p}$-diameter of $\mathbf{B}$ is

$$
\operatorname{diam}_{p}(B)=\max \left(2,2 d^{1 / p-1 / 2}\right)= \begin{cases}2 d^{1 / p-1 / 2} & \text { for } 1 \leq p \leq 2, \\ 2 & \text { for } 2 \leq p \leq \infty\end{cases}
$$

A.3. Spherical caps. Let $B=B_{2}^{d}(r)$ be the Euclidean ball of radius $r$, centered at the origin in $\mathbb{R}^{d}$. Let $C$ be a spherical cap of $B$ of height $h$, with $0 \leq h \leq r$; for instance,

$$
C=\left\{\left(x_{1}, \ldots, x_{d}\right) \in B: r-h \leq x_{1} \leq r\right\} .
$$

The volume of $C$ can be determined exactly, but the precise formula is awkward for large $d$ (one has to evaluate the integral $\int \sin ^{d} \theta d \theta$ ). On the other hand, we can easily obtain a lower bound for $\operatorname{vol}(C)$ by inscribing in it a "hypercone" $H$ of height $h$ whose base is a $(d-1)$-sphere of radius $s=\sqrt{r^{2}-(r-h)^{2}}=\sqrt{2 r h-h^{2}}$. For $r-h \leq x \leq r$, the cross-section of $H$ at $x=x_{1}$ is $B_{2}^{d-1}(s(r-x) / h)$, so applying (14) gives

$$
\operatorname{vol}(H)=\frac{2^{d-1} s^{d-1} \Gamma\left(\frac{3}{2}\right)^{d-1}}{h^{d-1} \Gamma\left(\frac{d+1}{2}\right)} \int_{r-h}^{r}(r-x)^{d-1} d x
$$


Random geometric graph diameter in the unit ball (with Robert B. Ellis and Catherine Yan) Algorithmica 47, no. 4 (2007), $421--438$. The original publication is available at www.springerlink.com: http://dx.doi.org/10.1007/s00453-006-0172-y Open Access version: http://kuscholarworks.ku.edu/dspace/.
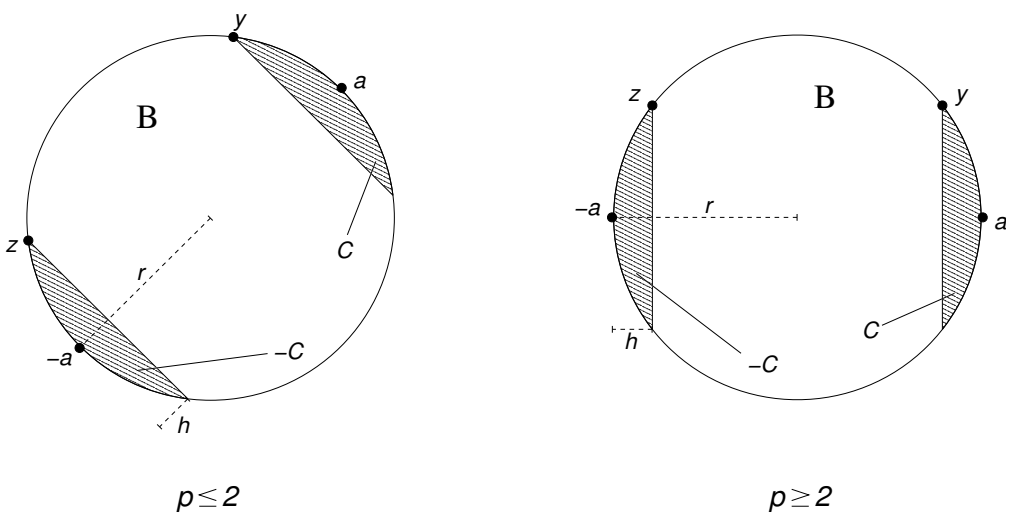

FiguRE 4. Antipodally aligned caps $\pm C$ centered at antipodes $\pm a$ of $B \subseteq \mathbb{R}^{2}$ for different values of $p$ below and above 2 .

$$
=\frac{\pi(2 r-h)^{(d-1) / 2} h^{(d+1) / 2}}{d \cdot \Gamma\left(\frac{d+1}{2}\right)} .
$$

Since $2 r-h \geq r$, we have the bound

$$
\operatorname{vol}(C) \geq \frac{\pi r^{(d-1) / 2}}{d \cdot \Gamma\left(\frac{d+1}{2}\right)} \cdot h^{(d+1) / 2} .
$$

A.4. The $\ell_{p}$-distance between opposite spherical caps. By definition, the $\ell_{p}$-distance between two sets $Y, Z \subseteq \mathbb{R}^{d}$ is

$$
d_{p}(Y, Z):=\inf \left\{\|y-z\|_{p}: y \in Y, z \in Z\right\} .
$$

Let $B=B_{2}^{d}(r)$, let $\pm a$ be a pair of $\ell_{p}$-antipodes on $B$, and let $\pm C$ be the cap of height $h$ centered at $\pm a$. Note that every pair $y, z$ at minimum distance has displacement parallel to $a$ (see Figure 4). (This can be verified by another easy Lagrange-multiplier calculation.) Therefore

$$
d_{p}(C,-C)=\frac{2(r-h)}{r}\|a\|_{p}= \begin{cases}2(r-h) d^{1 / p-1 / 2} & \text { for } 1 \leq p \leq 2 \\ 2(r-h) & \text { for } p \geq 2\end{cases}
$$

\section{REFERENCES}

[1] Alon, N., Spencer, J.H.: The probabilistic method. 2nd edn. John Wiley (2000)

[2] Bollobás, B.: Random Graphs. 2nd edn. Cambridge University Press (2001)

[3] Conway, J.H., Sloane, N.J.A.: Sphere Packing, Lattices and Groups. 3rd edn. Springer-Verlag (1998)

[4] Chen, X., Jia, X.: Package routing algorithms in mobile ad-hoc wireless networks. In: 2001 International Conference on Parallel Processing Workshops. (2001) 485-490

[5] Ellis, R.B., Jia, X., Yan, C.H.: On random points in the unit disk. Random Structures Algorithms (to appear)

[6] Ellis, R.B.: Approximating $\ell_{p}$-metrics with path distance in a random geometric graph. (preprint)

[7] Godehardt, E., Jaworski, J: On the connectivity of a random interval graph. Random Structures Algorithms 9 (1996) 137-161

[8] Penrose, M.D.: On $k$-connectivity for a geometric random graph. Random Structures Algorithms 15 (1999) 145-164

[9] Penrose, M.D.: Random Geometric Graphs. Oxford University Press (2003) 
Random geometric graph diameter in the unit ball (with Robert B. Ellis and Catherine Yan) Algorithmica 47, no. 4 (2007), $421--438$. The original publication is available at www.springerlink.com: http://dx.doi.org/10.1007/s00453-006-0172-y Open Access version: http://kuscholarworks.ku.edu/dspace/.

[10] Stojmenovic, I., Seddigh, M., Zunic, J.: Dominating sets and neighbor elimination-based broadcasting algorithms in wireless networks. IEEE Trans. Parallel Distrib. Syst. 13 (2002) $14-25$

[11] Whittaker, E.T., Watson, G.N.: A course of modern analysis. Cambridge University Press (1996)

[12] Wu, J., Li, H.: A dominating-set-based routing scheme in ad hoc wireless networks. Telecommunication Systems 18 (2001) 13-36

Department of Applied Mathematics, Illinois Institute of Technology, Chicago, IL 60616, USA

E-mail address: rellis@math.iit.edu

Department of Mathematics, University of Kansas, Lawrence, KS 66045-7523

E-mail address: jmartin@math.ku.edu

Department of Mathematics, Texas A\&M University, College Station, TX 778433368, USA

E-mail address: cyan@math.tamu.edu 